\title{
Heart transplantation in the treatment of primary non-operable cardiac tumors
}

\author{
Uladzimir U. Andrushchuk ${ }^{1}$, Youry P. Ostrovsky ${ }^{1}$, Andrei V. Valentsiukevich ${ }^{1}$, Liana G. Shestakova ${ }^{1}$, \\ Siarhei G. Amelchanka ${ }^{1}$, Valery G. Krutau ${ }^{1}$, Olga A. Yudina ${ }^{2}$, Pavel F. Chernoglaz ${ }^{1}$, Irina I. Grinchuk ${ }^{1}$, \\ Andrei Smalenski \\ ${ }^{1}$ Department of Cardiac Surgery, Republican Scientific Practical Centre "Cardiology", Minsk, Belarus \\ 2Pathology Department, Health Care Institution "City Clinical Pathology Bureau”, Minsk, Belarus
}

Kardiochirurgia i Torakochirurgia Polska 2017; 14 (4): 271-279

Tumor resection within healthy tissues is the main treatment method for patients with primary benign cardiac tumors, which account for up to $90 \%$ of all primary cardiac tumors [1-3]. Short-term and long-term results after such interventions are generally good, and the 10-year recurrence-free survival is about $93-100 \%[3,4]$. Even in the case of incomplete resection of a benign cardiac tumor because of its size and localization (mostly in large intramural ventricle tumors), the prognosis is still good, because these tumors grow slowly and are not associated with a systemic adverse influence [5]. Patients with non-resectable benign cardiac tumors may be candidates for orthotopic heart transplantation $(\mathrm{OHT})[6,7]$.

Primary malignant tumors, of which $75 \%$ are sarcomas [8], are commonly diagnosed at an advanced stage, because of rapid growth; and only in $15 \%$ of patients is it possible to perform tumor resection, and this without significant prognosis improvement [8]. In the natural disease course average survival time in this category of patients is about 10 months after diagnosis [8, 9]. Polychemotherapy (PCT) and radiotherapy (RT) do not provide improvement of long-term survival [10]. Extended resections of tumorinvolved cardiac structures and reconstructive interventions with heart autotransplantation (HA) in combination with neo-adjuvant and adjuvant PCT and RT in selective cases may improve long-term survival in patients with primary malignant tumors of atria, and to a lesser extent in patients with involvement of ventricles [11]. Local tumor recurrence even after pathology-proven radical resection (RO) is the main cause of mortality in the early and midterm postoperative follow-up period [2, 3, 6]. Until now OHT has rarely been performed in patients with primary non-resectable malignant cardiac tumors because of controversial results achieved $[6,12,13]$, because of donor-heart deficit and because of the legislation in several countries against performing such interventions to treat this pathology [3]. However, there is still a certain interest in this intervention as a method of treatment for primary malignant cardiac tumors, because of separate successive examples [14] and the absence of effective alternative treatment. In this report we present our experience in performing OHT for primary benign and malignant cardiac tumors.

Two patients (1 male and 1 female) with non-resectable cardiac tumors were surgically treated at SI RSPC Cardiology of Republic of Belarus during the period from May 2011 to May 2015. In one case the tumor involving the right ventricle (RV) and interventricular septum (IVS) was benign, and in the remaining case the tumor was malignant, involving all cardiac chambers. In these 2 patients OHT was performed. Interventions were performed according to previously described biatrial and bicaval methods [15] under normothermic perfusion. Custodiol solution was used for cardioplegia. Mid-term results were obtained during repeated in-hospital examination of patients.

Case 1: A 49-year-old female patient referred to the hospital in March, 2011 complaining of dyspnoea on moderate physical exertion and first appearance of syncopal condition. At the age of 24 she was diagnosed with a tumor of the IVS with a size of $15 \mathrm{~mm}$ according to transthoracic echocardiography (EchoCG) data. The patient was followed up with annual examinations and felt well until recently, despite slow tumor growth (Fig. 1). Non-invasive slow tumor growth, absence of signs of distant metastases according to computed tomography (CT), other clinical signs, and the patient's feeling of well-being all suggested benign tumor. Multiple episodes of paroxysmal ventricular tachycardia were registered in hospital settings, and were successfully managed with medicinal treatment and cardioversion. Transthoracic and transoesophageal EchoCG revealed a neoplasm $47 / 27 \mathrm{~mm}$ in the middle and lower third of the IVS and RV containing calcification foci. The neoplasm restrained the right ventricular outflow tract with peak pressure gradient of $18 \mathrm{~mm} \mathrm{Hg}$. Right ventricle and left ventricle (LV) contractility, dimensions of cardiac cavities and func-

Address for correspondence: Dr. Siarhei G. Amelchanka, Department of Cardiac Surgery, Republican Scientific Practical Centre “Cardiology”, 110 R. Luxemburg St, 220036 Minsk, Belarus, phone: +37 5297848684, e-mail: omelchenkosergey2@gmail.com Received: 30.06.2017, accepted: 21.08.2017. 


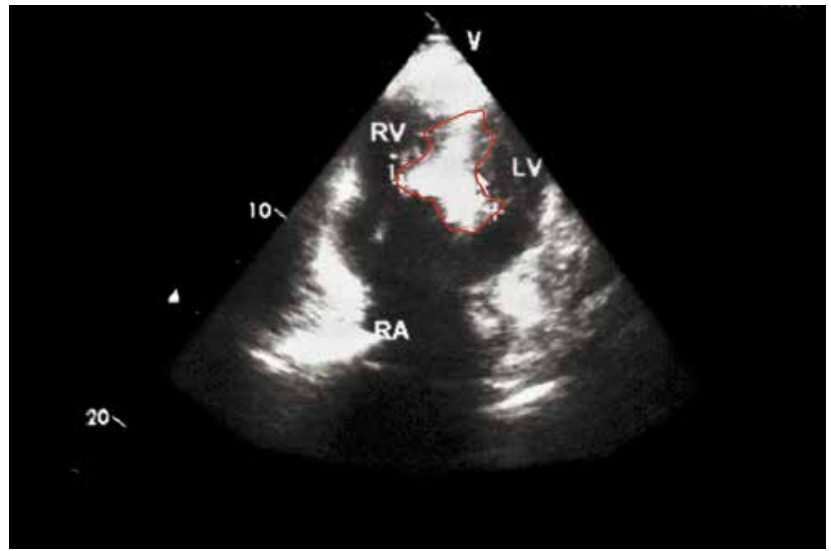

Fig. 1. Tumor of interventricular septum and right ventricle of $40 / 20 \mathrm{~mm}$ (EchoCG dated 2009). The visible part of the tumor is demarked by a red line

$\mathrm{RV}$ - right ventricle, RA - right atrium, LV - left ventricle

tion of heart valves were within the normal range. On day 5 a cardioverter-defibrillator (ICD) Medtronic Maximo II DR was implanted in the patient, she received 3-day preventive antibiotic treatment and on day 10 she was discharged in a satisfactory condition. Eleven days later the patient was hospitalized again complaining of exertional dyspnea, low-grade fever, right arm edema with right hand trophic skin lesion, paroxysms of increased heart rate and multiple episodes of ICD triggering. Ultrasound angio-investigation showed thrombophlebitis of the right internal jugular vein and subclavian veins with partial obstruction, and CT data showed left lower lobe pneumonia with moderate hydrothorax. During a 3-week period the patient received the following therapy: antibiotics, cardiotropic and antiarrhythmic therapy, angioprotective therapy, antiplatelets, anticoagulant and diuretic therapy; she was put on the waiting list for heart transplantation and investigated according to the respective protocol, and later was discharged in a satisfactory condition. On 26.05.2011 OHT was performed in the patient using the standard biatrial method, and the

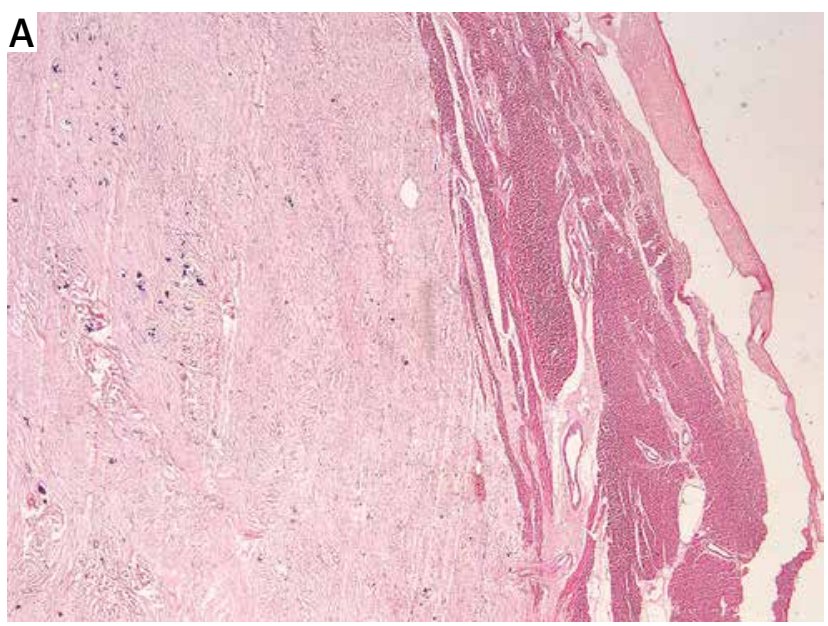

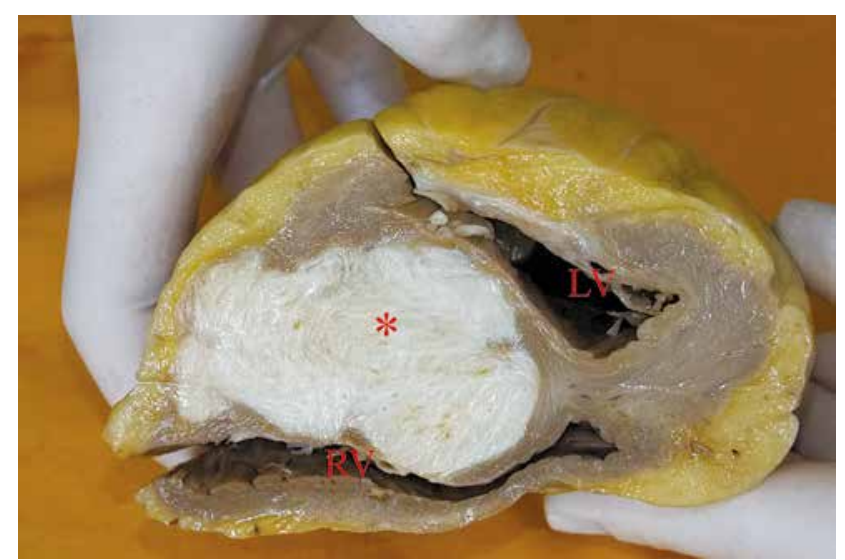

Fig. 2. Gross specimen of the recipient heart containing benign tumor (leiomyoma) on the cross-sectioned surface: tumor is indicated by asterisk

$\mathrm{RV}$ - right ventricle, $\mathrm{LV}$ - left ventricle

ICD was explanted. Duration of donor heart ischemia was 180 min. An intra-aortic balloon counterpulsation (IABC) system was used before disconnecting the patient from the cardiopulmonary bypass because of left ventricular insufficiency refractory to cardiotonic support. A cross-sectional macroscopic specimen of the recipient's explanted heart with the tumor is presented in Figure 2. Major in-hospital complications were registered in the postoperative period: cardiac tamponade, intrapericardial hemorrhage, multiple organ failure (cardiac, renal, respiratory), sepsis (blood cultures of Enterococcus faecalis and Acinetobacter baumannii were isolated), and serofibrinous mediastinitis. In total the patient underwent five resternotomy interventions: on the second postoperative day cardiac decompression was performed and central veno-arterial extracorporeal membrane oxygenation (ECMO) was established; on the fourth postoperative day removal of blood clots from the mediastinum was performed; on the eighth postoperative day control of bleeding in the area of aortic ECMO was performed (resulting from cannula perforation) with cannula replacement; on

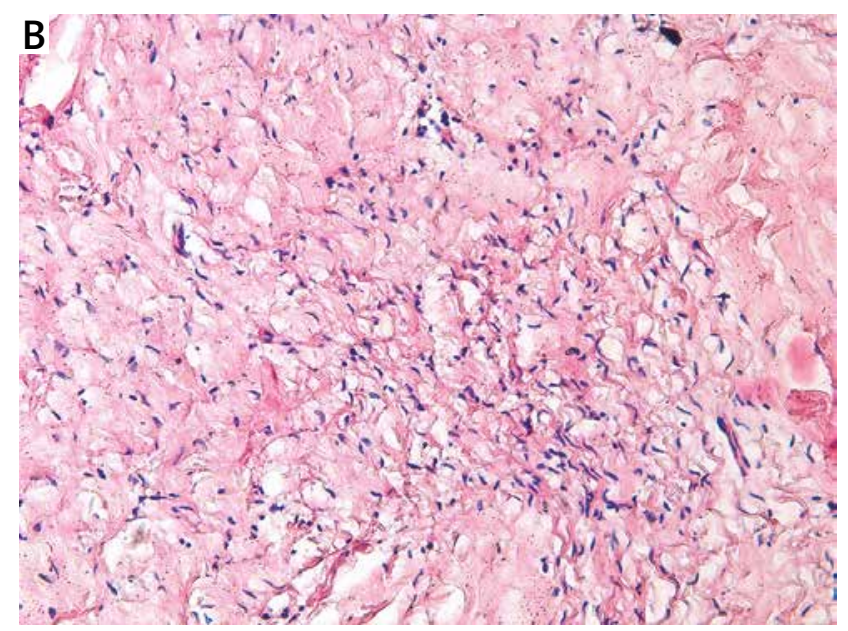

Fig. 3. Recipient heart tumor pathology study: leiomyoma: A - Thin strip of RV myocardium on the right side of the figure. The tumor has severe spreading secondary changes: sclerosis and small foci of calcification. $\mathrm{H}+\mathrm{E}, 1.25 \times$ objective. $\mathrm{B}-$ The tumor is represented by fibers and cells with regular elongated nuclei without polymorphism, with $\alpha$-SMA-positive immunohistochemistry. $\mathrm{H}+\mathrm{E}, 1.25 \times$ objective 
the eleventh postoperative day central ECMO was discontinued; on the $33^{\text {rd }}$ day repeated sternotomy, upper median laparotomy, omentomediastinopexy, and repeated sternal osteosynthesis were performed. On the seventh day the IABC system was removed. During the first 14 days after the OHT the patient was on continuous renal replacement therapy in hemodialysis mode. Three agents were used for immune suppression: tacrolimus, mycophenolate mofetil, prednisolone. Intensive cardiotropic therapy, antibacterial therapy and other drug therapies were used according to the protocol after $\mathrm{OHT}$ and to treat complications. The patient was discharged in a satisfactory condition on the $103^{\text {rd }}$ day after the OHT. Four years and 8 months after the intervention the patient felt well, had no complaints, EchoCG parameters were within the normal range, she had no signs of perfusion disturbances in brachiocephalic and jugular veins according to Doppler ultrasound data, and the 6-minute walk test showed $580 \mathrm{~m}$. The pathology study confirmed the presence of a benign tumor - leiomyoma (Fig. 3).

Case 2: A male patient aged 62 years in a severe condition was referred on August, 2013, to RSPC Cardiology by medical transport from the Oncology Centre. On admission the patient complained of dyspnea at rest, and swelling of the ankles and feet. In 1996 the patient was diagnosed with coronary artery disease, and subsequently coronary artery angioplasty was performed. Three months before hospitalization dyspnea appeared in the patient with multiple syncopal episodes. The patient was investigated in a regional hospital institution, where electrocardiography revealed third degree
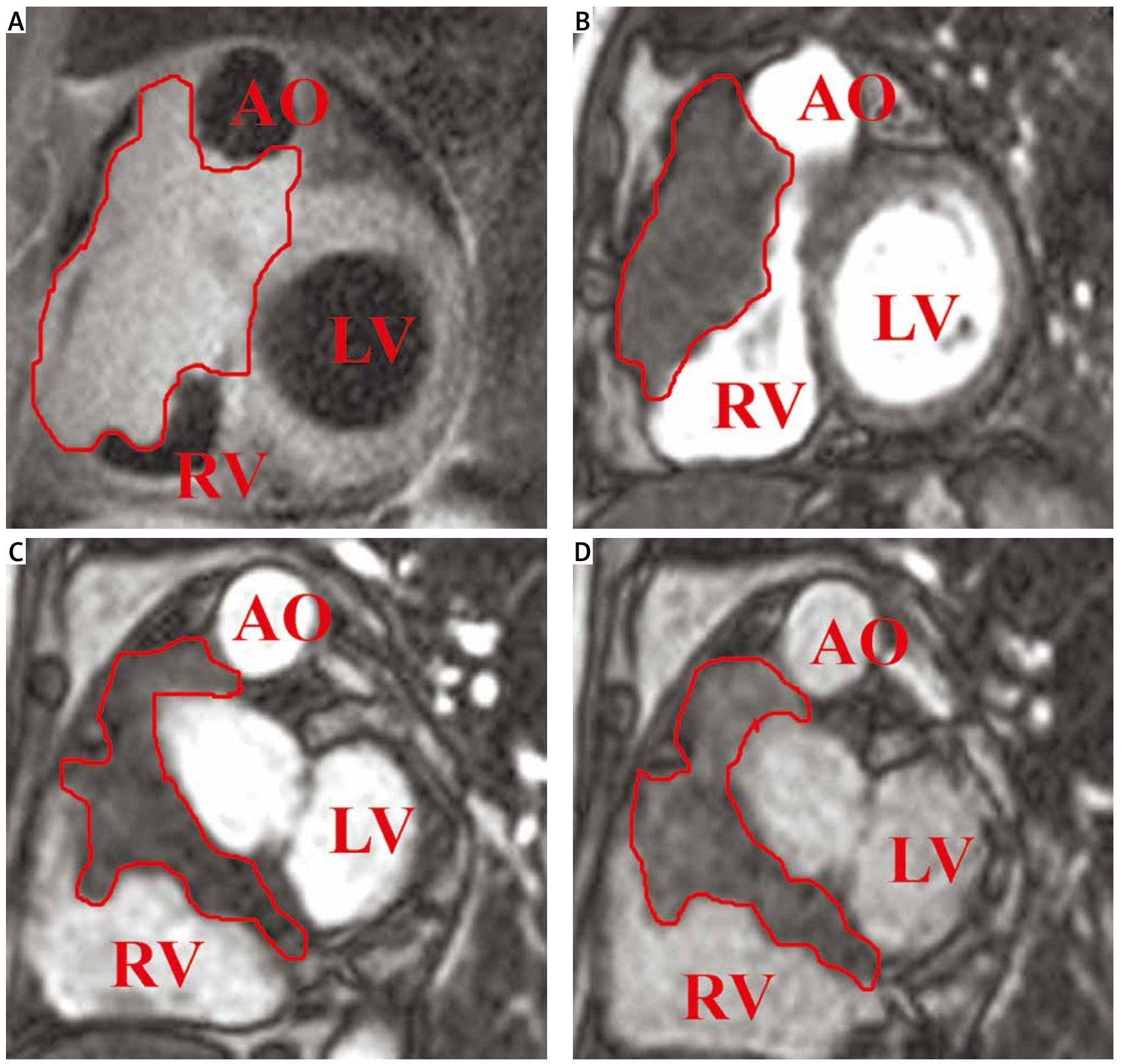

Fig. 4. Right ventricle tumor spreading to right and left atrium, and to aortic root (MRI)

$\mathrm{LV}$ - left ventricle, AO - aorta, RV - right ventricle 
transient atrioventricular block, and transthoracic EchoCG showed a right atrial tumor measuring $55 / 75 \mathrm{~mm}$ of irregular shape (without significant contractility disturbances) spreading to the interatrial septum (IAS), subsequently confirmed by magnetic resonance imaging (MRI) (Fig. 4). Coronarography showed no significant coronary artery pathology. An electric cardiac pacemaker (Biotronic Effecta DR) was implanted in the patient on May, 2013, and he was diagnosed with right
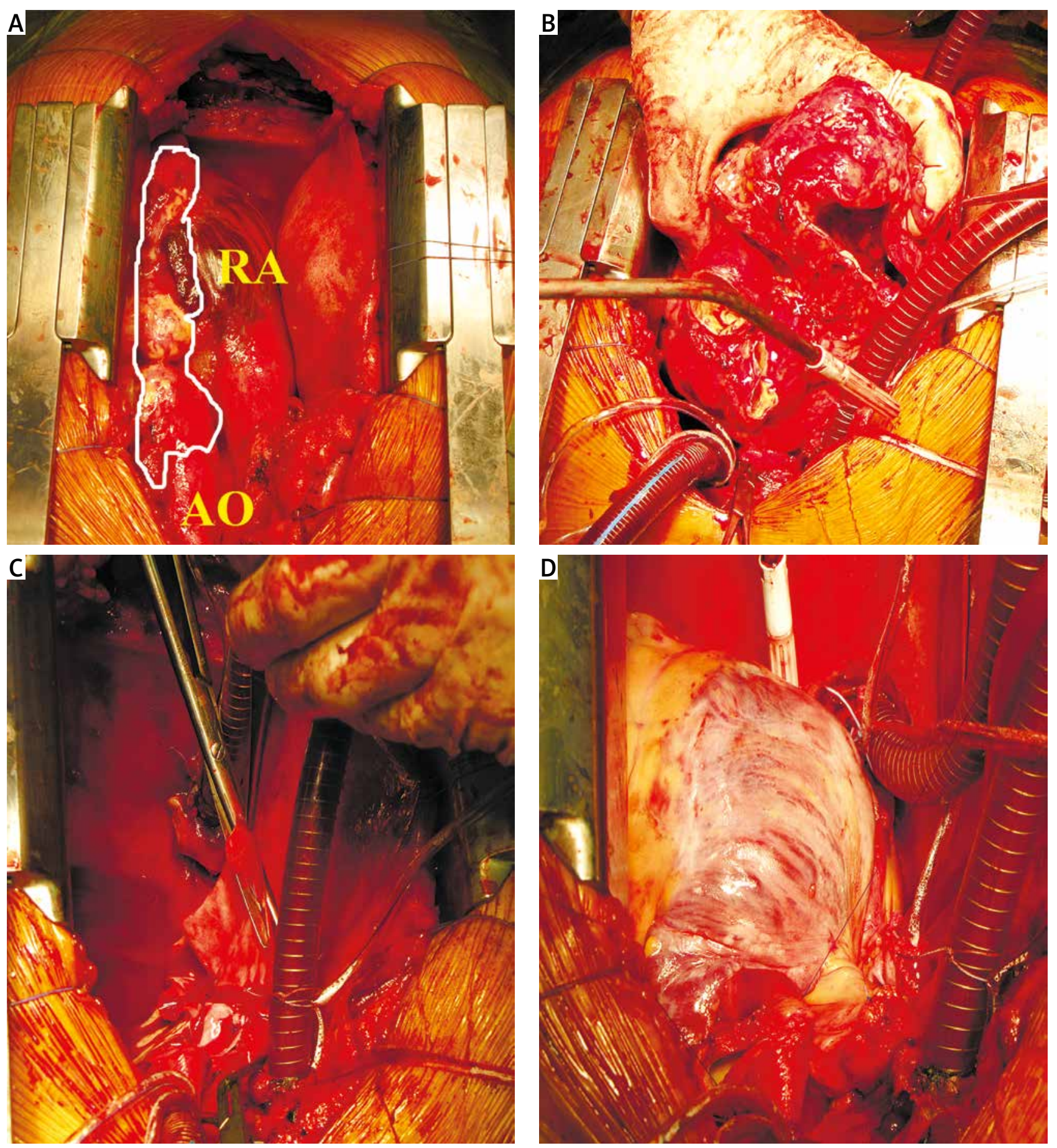

Fig. 5. Orthotopic heart transplantation steps in patient with primary non-respectable malignant tumor of the heart: A - heart tumor revision after sternotomy. The visible part of the tumor is demarked by a white line. B - Explantation of the recipient heart with tumor. C - Reconstruction of neo-atrium using xenopericardium. D - Completion of orthotopic heart transplantation

$\mathrm{RA}$ - right atrium, $\mathrm{AO}$ - aorta 
racic EchoCG performed at RSPC Cardiology; the tumor was characterized by irregular shape and rough margins; it was predominantly localized in the area of the right atrium (RA) and basal region of the RV, spreading to the IAS and LA. The neoplasm surrounded the aortic root on every side, caused partial of the tricuspid valve (effective orifice area: $1.5-1.8 \mathrm{~cm}^{2}$ ), and spread to the outflow tract of the right ventricle and initial portion of the pulmonary artery. The tumor also invaded the cusps of the tricuspid and mitral valves, causing second and third degree regurgitation, respectively. Antero-posterior dimension of the RV was 47/87 mm in 4-chamber view, RA 55/69 mm, LV ejection fraction was $49 \%$ with diastolic dysfunction of restrictive type, mean pulmonary artery pressure was $30 \mathrm{~mm} \mathrm{Hg} .380 \mathrm{ml}$ of fluid were visualized in the pericardial cavity. Abdominal ultrasound data and gastric endoscopic study showed no pathology. Laboratory investigations: NTproBNP 5248 pg/ml, procalcitonin $6.09 \mathrm{ng} / \mathrm{ml}$, CRP $48.3 \mathrm{mg} / \mathrm{l}$. Percutaneous transvenous tumor biopsy was considered inappropriate because of the patient's severe condition. We had no opportunity to perform positron emission tomography. Orthotopic heart transplantation upon availability of a matching-heart donor was chosen as a method of acute treatment, where intraoperative tumor revision is supposed to be performed first, after which in case of distant metastases or tumor invasion into other organs the heart is supposed to be transplanted to an alternative recipient. On August, 2013 OHT and removal of the cardiac pacemaker were performed. After pericardiotomy no tumor invasion into other mediastinal organs was revealed nor tumor dissemination. The recipient's heart was explanted together with the affected aortic root, and a portion of the ascending aorta (about $3 \mathrm{~cm}$ ), heart-adjacent portions of the superior (SVC) and inferior vena cava (IVC), a major portion of the LA posterior wall, leaving separately 2 right pulmonary veins and one tissue site containing left pulmonary veins. The LA posterior wall was repaired using a xenopericardium patch $10 \times 10 \mathrm{~cm}$, and to repair the IVC portion another patch $(3 \times 4 \mathrm{~cm})$ was used, with subsequent OHT according to bicaval technique [15] - Fig. 5 . Donor heart myocardial ischemia duration was 157 min, cardiopulmonary bypass (CPB) duration was 224 min. Cardiac function restored after one defibrillation. Thorough hemostasis was performed due to significant diffuse tissue bleeding. Inotropic support after cardio-pulmonary bypass completion: dobutamine $3.5 \mu \mathrm{g} / \mathrm{kg} / \mathrm{min}$, Levonor $0.1 \mu \mathrm{g} / \mathrm{kg} / \mathrm{min}$ (norepinephrine bitartrate). Total duration of procedure was $510 \mathrm{~min}$, intraoperative blood loss was $1350 \mathrm{ml}$ (red blood cells autoreinfusion - $550 \mathrm{ml}$ ). Postoperative blood loss - $250 \mathrm{ml}$. Signs of acute liver, renal and moderate cardiac failure were registered in the early postoperative period (total bilirubin $151 \mu \mathrm{mol} / \mathrm{l}$, direct bilirubin $65 \mu \mathrm{mol} / \mathrm{l}$, AST $700 \mathrm{U} / \mathrm{l}$ and GFR $0.308 \mathrm{ml} / \mathrm{s} / 1.73 \mathrm{~m}^{2}$ ). Standard triple drug immunosuppressive therapy (tacrolimus, mycophenolate mofetil, prednisone) and early rejection prophylaxis with anti-thymocyte globulin at a dose of $1.25 \mathrm{mg} / \mathrm{kg}$ over 2 days were used. Continuous ultrahemodi- afiltration was started $9 \mathrm{~h}$ after the surgical intervention. At that time a significant difference in central venous pressure between the superior and IVC was revealed, and stenosis of the IVC in the area of the anastomosis and implanted RA patch was suspected, which was confirmed by angiographic study showing $90 \%$ stenosis of the anastomotic site. Successful stent implantation into the anastomosis between the IVC and RA was performed (Fig. 6). Four days later renal replacement therapy was discontinued after renal function recovery. Recovery of consciousness, adequate spontaneous ventilation and muscle tone were achieved on day 7 , with subsequent extubation. Nevertheless, until day 12 there were signs of hepatic insufficiency (total bilirubin up to $390 \mu \mathrm{mol} / \mathrm{l}$, direct bilirubin up to $166 \mu \mathrm{mol} / \mathrm{l})$, cardiac (epinephrine $1 \mathrm{mg} / \mathrm{kg} / \mathrm{min}$, norepinephrine $2 \mathrm{mg} / \mathrm{kg} / \mathrm{min}$ ) and respiratory failure secondary to emerging pulmonary edema, requiring repeated intubation and mechanical ventilation. Cardiac arrest and unsuccessful CPR occurred on day 13. Pathology study of the recipient heart (Fig. 7): suspicion of large B-cell lymphoma of the heart involving major parts of the RA, RV, a portion of the LA, basal portions of the LV and MV, and with aortic root involvement. Donor heart resection margins contained no signs of tumor growth. Autopsy confirmed patency of anastomosis between recipient IVC and RA of the donor heart in the area of stent placement; pulmonary edema and extensive hepatocellular necrosis were also confirmed by autopsy (Fig. 8); no regional or distant metastases were found.

In patients with non-resectable benign cardiac tumors (with significant myocardium damage, spreading to the cardiac conduction system and with involvement of cardiac valves, etc.), even partial tumor resection provides a positive long-term clinical effect, improving long-term survival [3-5], especially in adults [5]. However, in case of life-threatening conditions, unrelated to tumor blood

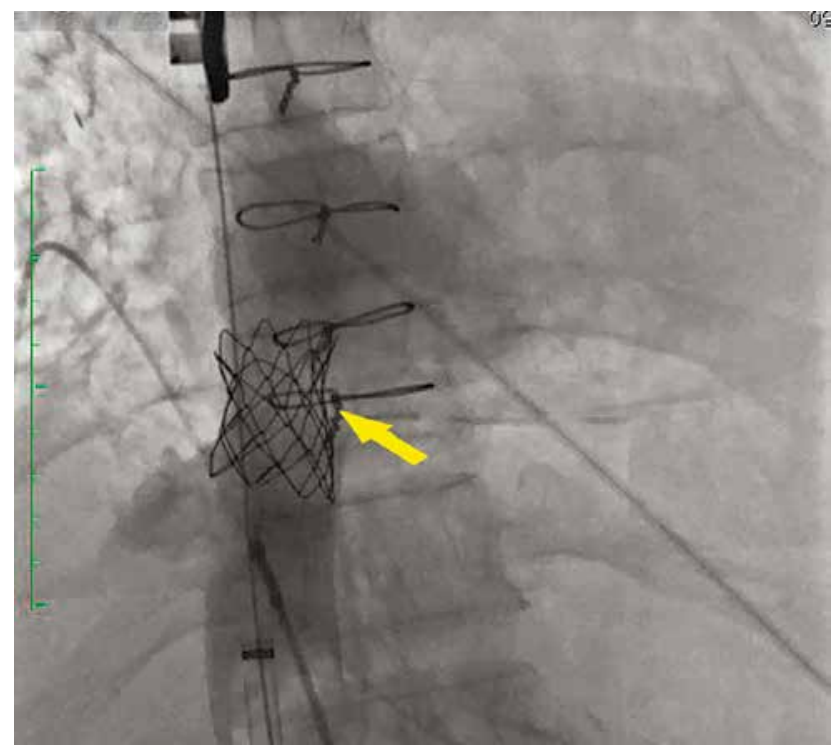

Fig. 6. Results of stent placement in the anastomotic area between vena cava inferior and right atrium - patency in the stenosis area was restored (cavography): stent is shown by the arrow 

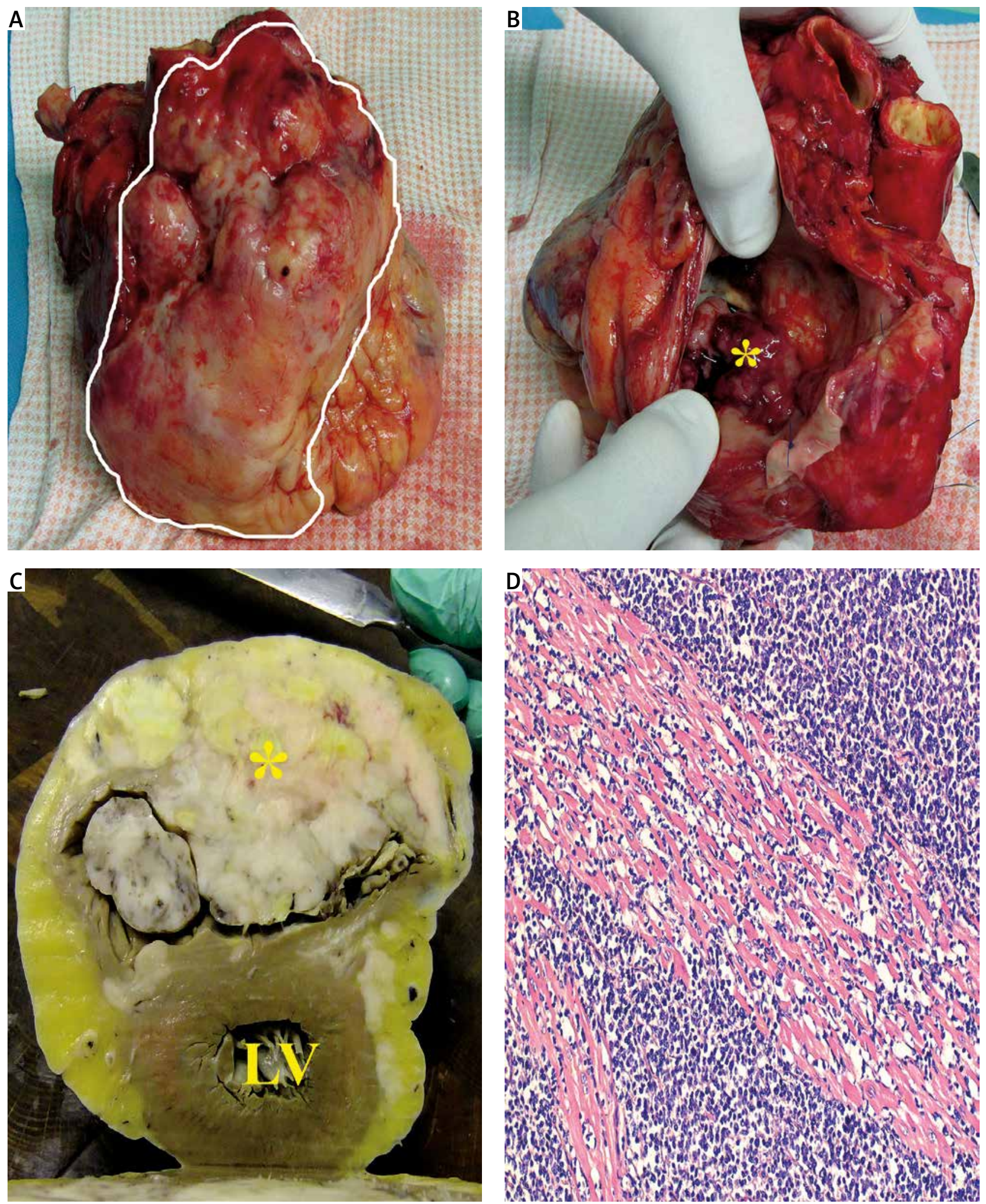

Fig. 7. Pathology study of the recipient heart, affected by lymphoma: A - gross specimen of the recipient heart - tumor is demarked by a white line. B - Mitral valve damaged by lymphoma (asterisk). C - Fixed gross specimen of the heart (cross-section under the line of atrioventricular valves fibrous rings). Tumor (asterisk) presented by white foci replaces the myocardium of the right ventricle, invades epicardium, grows around coronary arteries, diffusely spreading in the myocardium of the left ventricle (LV). D - Microscopic picture of large B-cell lymphoma of the heart. Tumor replaces the myocardium and invades interstitium between muscle cells; tumor is presented by solid areas containing polymorphic round cells with positive immunohistochemistry for CD 20. 200x objective, H + E staining 

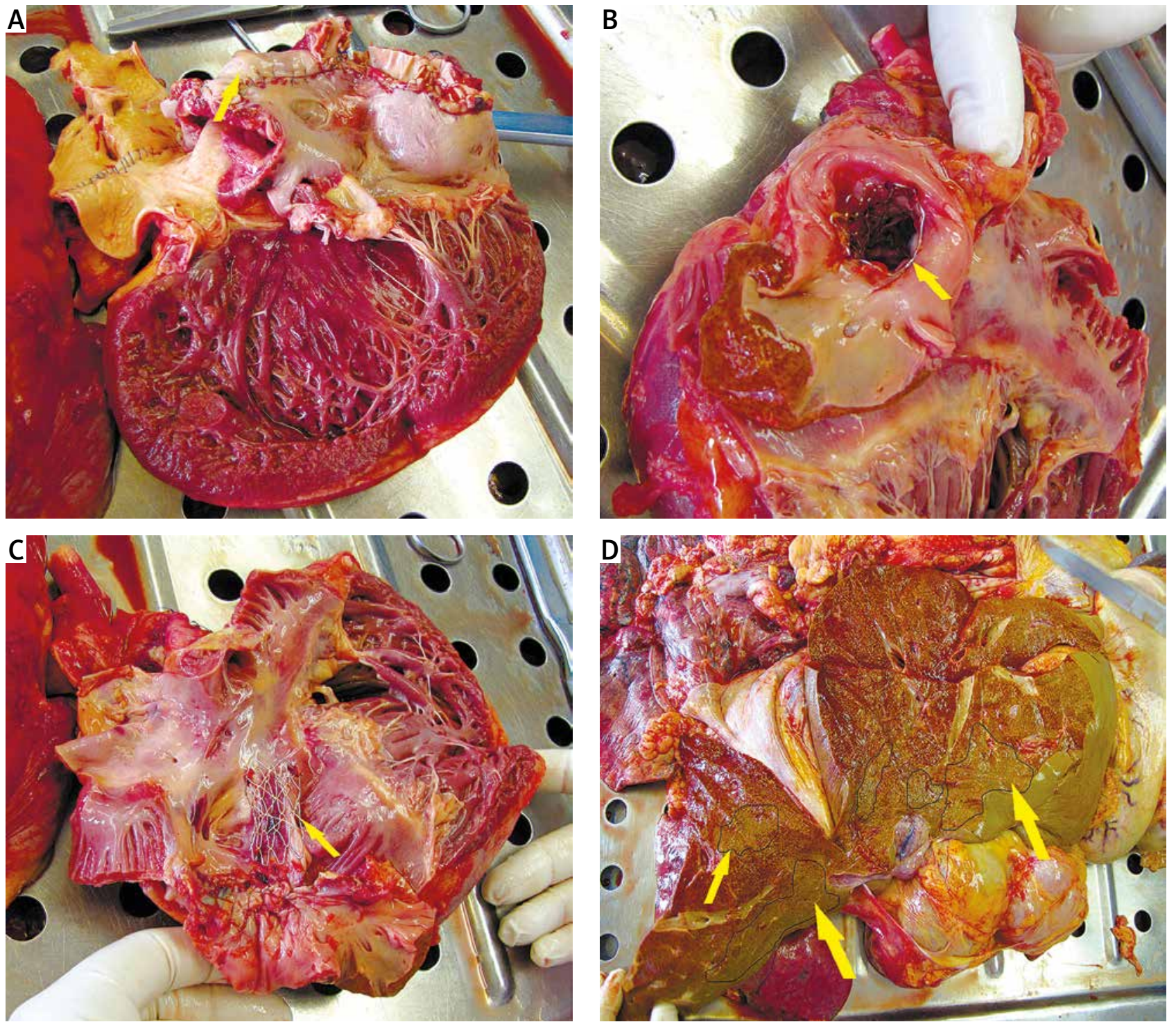

Fig. 8. Autopsy results: A - donor heart with repaired left atrium using xenopericardium patch. Patch is shown by arrow. $\mathbf{B}$ and $\mathbf{C}-\mathrm{Pa}$ tency of anastomosis between IVC and RA of the donor hear in the area of stent placement, stent is shown by arrow. D - Extensive haemodynamic necrosis (yellow areas - arrow)

flow obstruction, but due to difficult cardiac rhythm disturbances (frequent paroxysmal ventricular tachycardia, ventricular fibrillation) or decreased ventricular contractility resulting from intramural benign tumor, its partial resection is usually not associated with any clinically significant benefit. We suggest that OHT can be a method of choice in particular for these rare benign cardiac tumors. Shortterm and long-term results of such interventions usually are good and excellent, even in patients with a complicated course of the disease $[12,16]$. In our case large intramural leiomyoma of the IVS and RV caused not only partial tricuspid valve and RV outflow tract obstruction, but also frequent ventricular tachycardia paroxysms, requiring ICD implantation, with subsequent thrombophlebitis of the right internal jugular and subclavian vein with trophic disturbances of the right wrist. Severe baseline status of the patient defined to a large extent multiple in-hospital peri- operative complications. Nevertheless, the long-term result can be regarded as good.

In contrast to benign tumors, only $15 \%$ of primary malignant cardiac tumors are resectable at the time of diagnosis, because of rapid invasive growth [17]. The principal difficulty in determining indications for intervention in patients with primary malignant cardiac tumors resides in the necessity to confirm the absence of extracardiac tumor spread. All up-to-date techniques are used to investigate these patients including positron emission tomography (PET) [18] PCT and RT alone do not provide survival improvement in patients with malignant cardiac tumors $[10,17]$. Survival time in patients after malignant cardiac tumor resection is also relatively short, and usually is 1 to 2 years [2, 3], and only in isolated cases reaches 3 to 4 years [4, 5]. The best results in cases of unresectable tumors are achieved after tumor resection in combination with adjuvant PCT 
and RT $[4,17]$. However, even in rare cases of radical tumor resection (RO) local recurrence of primary malignant cardiac tumor is the leading cause of mortality in the early and midterm follow-up period, and is the main indication for repeated intervention $[3,4,7]$. Heart autotransplantation allows one in individual cases to increase the portion of resectable tumors and to improve the prognosis [11], but it mainly refers to LA tumors. Heart autotransplantation benefit is currently recognized for cases of atrial malignant tumors without invasion of pulmonary veins and without necessity of pulmonary resection. Otherwise the rate of major hospital complications is high, and early and midterm survival is low $[11,17]$. Whereas OHT frequency in malignant tumors is higher (75\%) than in benign tumors, the controversial results achieved make many surgeons have doubts regarding this treatment approach $[6,12,13]$. According to international recommendations [19] OHT is not contraindicated in malignant cardiac tumors with a low expected recurrence rate, based on tumor type, response to treatment and absence of distant metastases according to investigations results (class I, level C). It was also noted that the time-lag between remission induction and the possibility of heart transplantation depends on the abovementioned factors, and this time period was not exactly determined. On the other hand, deficit of donor organs, possible influence of immunosuppressive therapy on recurrence rate and tumor progression after the intervention, prohibition of OHT for primary cardiac malignant tumors in several countries [3], and largely unsatisfactory results $[2,6,20]$ until today significantly limit the use of this treatment method. Tumor progression (local recurrence and distant metastases) is the cause of death in the early and midterm period in such patients. According to an analysis of 28 heart transplantations for primary cardiac tumors (7 benign and 21 malignant), performed in 2000 [13], mean survival after transplantation for benign tumors was 46 months, and for malignant tumors it was 12 months. Even in a series of 4 heart-lung complex transplantations the results were unsatisfactory because of tumor progression in all the patients - mean survival time was 31 months (5-49 months) [9]. Our study confirmed the necessity not only for local tumor control with surgical treatment methods, but also for improvement of systemic control of tumor progression using up-to-date PCT and RT regimens. On the other hand, today the true value of PCT (especially against immunosuppression) and RT after OHT is still uncertain and requires further investigation [13]. However, today there is no other effective alternative to OHT in combination with PCT and RT for primary non-resectable cardiac tumors. Cautious optimism is inspired by isolated successful cases of OHT for primary malignant cardiac tumors - the best long-term survival time reported was 102 months [14]. Long-term results may also depend on the malignant tumor type [13], and treatment strategy may depend on cardiac tumor localization [17]. Treatment for right heart sarcomas (associated with early metastases) is initiated with neoadjuvant (4-6 courses) PCT; histological tumor type and indications for surgical treatment are then determined. Left heart sarcomas are more restrained, characterized by less infiltrative growth, and are associated with late metastases but early cardiac insufficiency. Commonly these are initially treated surgically because of cardiac failure [21]. The best results are achieved after OHT for primary malignant tumors, excluding angiosarcomas, which is the most aggressive sarcoma type [13], even after radical resection [12]. In order to increase the chances for radical resection (R0) the majority of authors [22] recommend using the bicaval method of OHT [15]. Some authors consider tumor-induced life-threatening conditions one of the main indications for OHT for malignant (both primary and secondary) cardiac tumors [23]. Total artificial heart in patients with primary non-resectable malignant cardiac tumors is a promising future treatment method, requiring no immunosuppressive therapy and independent of donor heart availability. However, there is only a single case experience [24].

In our case presented OHT was performed in a patient in a critical condition, where the tumor affected all cardiac chambers, with almost total RV involvement, and with severe atrioventricular valve dysfunction. Transvenous myocardial biopsy is commonly used in right heart tumor diagnostics, and in some instances allows surgical intervention to be avoided [25]. However, in this case there was not sufficient time to perform an anthracycline- and anti-CD20-based drug therapy even after lymphoma was confirmed; therefore we refused biopsy. Some reports [12, 13] also describe OHT for primary malignant lymphomas of the heart on the background of tumor-induced emergency conditions. Our negative experience in OHT for primary malignant tumor of the heart was associated with extensive heart involvement (including IVC ostia) and a strategic intraoperative misstep, which led to subsequent severe hepatocellular necrosis. However, based on the absence of tumor signs in resection margins and the possibility of drug treatment, we suggest the possibility of survival time improvement in this case, provided the absence of perioperative complications.

Thus, today we cannot recommend OHT as a standard treatment method for benign and malignant non-resectable cardiac tumors. However, in a selective group of patients (especially in benign tumors), this treatment method could be beneficial.

In conclusion, OHT is a method of choice in a selected group of patients with non-resectable primary benign cardiac tumors, which are the cause of life-threatening conditions. Short-term and long-term results of this intervention are good. We should continue to research the role of heart transplantation in the treatment of a selected group of patients with primary non-resectable malignant cardiac tumors, based on tumor type and its localization, considering recent advances in polychemo- and radiotherapy, and also considering the absence of other effective alternatives.

\section{Disclosure}

Authors report no conflict of interest. 


\section{References}

1. Lobo A, Lewis JF, Conti R. Intracardiac masses detected by echocardiography: case presentation and review of the literature. Clin Cardiol 2000; 23: 702-708.

2. Centofanti P, Di Rosa E, Deorsola L, Dato GM, Patanè F, La Torre M, Barbato L, Verzini A, Fortunato G, di Summa M. Primary cardiac tumors: early and late results of surgical treatment in 91 patients. Ann Thorac Surg 1999; 68: 1236-1241.

3. Kamiya H, Yasuda T, Nagamine H, Sakakibara N, Nishida S, Kawasuji M, Watanabe G. Surgical treatment of primary cardiac tumors -28 years' experience in Kanazawa University Hospital. Jpn Circ J 2001; 65: 315-319.

4. Piazza N, Chughtai T, Toledano K, Sampalis J, Liao C, Morin JF. Primary cardiac tumours: eighteen years of surgical experience on 21 patients. Can J Cardiol 2004; 20: 1443-1448

5. Dein JR, Frist WH, Stinson EB, Miller DC, Baldwin JC, Oyer PE, Jamieson S, Mitchell RS, Shumway NE. Primary cardiac neoplasms: early and late results of surgical treatment in 42 patients. J Thorac Cardiovasc Surg 1987; 93: 502-511.

6. Jimenez Mazuecos JM, Fuentes Manso R, Segovia Cubero J, Toquero Ramos J, Oteo Dominguez JF, Alonso-Pulpon RL Is heart transplantation for primary cardiac sarcoma a useful therapeutic option? Rev Esp Cardiol 2003; 56: 408-411.

7. Vander Salm TJ. Unusual primary tumors of the heart. Semin Thorac Cardiovasc Surg 2000; 12: 89-100.

8. Straus R, Merliss R. Primary tumours of the heart. Arch Pathol 1945; 39: 74-80.

9. Talbot SM, Taub RN, Keohan ML, Edwards N, Galantowicz ME, Schulman LL Combined heart and lung transplantation for unresectable primary cardiac sarcoma. J Thorac Cardiovasc Surg 2002; 124: 1145-1148.

10. Llombart-Cussac A, Pivot X, Contesso G. Adjuvant chemotherapy for primary cardiac sarcomas: the IGR experience. Br J Cancer 1998; 78: 1624-1628.

11. Ramlawi B, Al-Jabbari O, Blau LN, Davies MG, Bruckner BA, Blackmon SH, Ravi V, Benjamin R, Rodriguez L, Shapira OM, Reardon MJ. Autotransplantation for the resection of complex left heart tumors. Ann Thorac Surg 2014; 98: 863-868.

12. Goldstein DJ, Oz MC, Rose EA, Fisher P, Michler RE. Experience with heart transplantation for cardiac tumors. J Heart Lung Transplant 1995; 14: 382-386.

13. Gowdamarajan A, Michler R. Therapy for primary cardiac tumors: is there a role for heart transplantation? Curr Opin Cardiol 2000; 15: 121-125.

14. Grandmougin D, Fayad G, Decoene C, Pol A, Warembourg H. Total orthotopic heart transplantation for primary cardiac rhabdomyosarcoma: factors influencing long-term survival. Ann Thorac Surg 2001; 71: 1438-1441.
15. Dreyfus G, Jebara V, Mihaileanu S, Carpentier AF. Total orthotopic heart transplantation: an alternative to the standard technique. Ann Thorac Surg 1991; 52: 1181-1184.

16. Michler R, Goldstein D. Treatment of cardiac tumors by orthotopic cardiac transplantation. Semin Oncol 1997; 24: 534-539.

17. Blackmon SH, Patel A, Reardon MJ. Management of primary cardiac sarcomas. Expert Rev Cardiovasc Ther 2008; 6: 1217-1222.

18. Inoue T, Koyama K, Oriuchi N, Alyafei S, Yuan Z, Suzuki H, Takeuchi K, Tomaru Y, Tomiyoshi K, Aoki J, Endo K. Detection of malignant tumors: whole-body PET with fluorine 18 alpha-methil tyrosine versus FDG. Preliminary study. Radiology 2001; 220: 54-62.

19. Mehra MR, Canter CE, Hannan MM, Semigran MJ, Uber PA, Baran DA, Danziger-Isakov L, Kirklin JK, Kirk R, Kushwaha SS, Lund LH, Potena L, Ross HJ, Taylor DO, Verschuuren EA, Zuckermann A; International Society for Heart Lung Transplantation (ISHLT) Infectious Diseases Council; International Society for Heart Lung Transplantation (ISHLT) Pediatric Transplantation Council; International Society for Heart Lung Transplantation (ISHLT) Heart Failure and Transplantation Council. The 2016 International Society for Heart Lung Transplantation listing criteria for heart transplantation: a 10-year update. J Heart Lung Transplant 2016; 35: 1-23.

20. Rodriguez Cruz E, Cintron-Maldonado RM, Forber TJ. Treatment of primary cardiac malignancies with orthotopic heart trasplantation. Bol Asc Medic 2000; 92: 65-71.

21. Blackmon SH, Patel A, Reardon MJ, Vaporciyan A, Reardon M. Right heart sarcomas. Methodist Debakey Cardiovasc J 2010; 6: 44-8.

22. Babatasi G, Massetti M, Agostini D, Galateau F, Saloux E, Nata P, Nataf $P$, Grollier G, Khayat A. Recurrent left-sided heart leiomyosarcoma: should heart transplantation be legitime. J Heart Lung Transplant 1998; 17: 1133-1138.

23. Agaimy A, Rösch J, Weyand M, Strecker T. Primary and metastatic cardiac sarcomas: a 12-year experience at a German heart center. Int J Clin Exp Pathol 2012; 5: 928-938.

24. Pirk J, Maly J, Szarszoi O, Urban M, Kotulak T, Riha H, Neuzil P, Netuka I. Total artificial heart support with two continuous-flow ventricular assist devices in a patient with an infiltrating cardiac sarcoma. ASAIO J 2013; 59: 178-180.

25. Abramowitz Y, Hiller N, Perlman G, Admon D, Beeri R, Chajek-Shaul T, Leibowitz $\mathrm{D}$. The diagnosis of primary cardiac lymphoma by right heart catheterization and biopsy using fluoroscopic and transthoracic echocardiographic guidance. Int J Cardiol 2007; 118: 39-40. 